\title{
Resonance crossing and dynamic aperture in nonscaling fixed field alternating gradient accelerators
}

\author{
Shinji Machida* \\ ASTeC, STFC Rutherford Appleton Laboratory, Harwell Science and Innovation Campus, Didcot, Oxfordshire, OX11 OQX, \\ United Kingdom
}

(Received 2 April 2008; published 25 September 2008)

\begin{abstract}
Operation with natural chromaticity in a linear nonscaling fixed field alternating gradient (FFAG) accelerator causes crossing of the low order resonances such as integer and half-integer. Although those resonances are not systematic ones, small errors, such as a typical misalignment of $10 \mu \mathrm{m} \mathrm{rms,}$ significantly increase particle amplitude when the accelerator is operated with a slow acceleration rate. For example, there is practically no dynamic aperture if it takes 1000 turns to finish the whole acceleration cycle. Chromaticity correction with sextupole and octupole reduces the maximum available dynamic aperture in a lattice without errors. On the other hand, the accelerator becomes less sensitive to errors. To use a nonscaling FFAG for applications where, unlike a muon accelerator, the large acceptance is not a high priority demand (such as a proton driver or a particle therapy accelerator), chromaticity correction seems to be an essential ingredient.
\end{abstract}

DOI: 10.1103/PhysRevSTAB.11.094003

PACS numbers: 29.20.-c, 41.85.-p, 29.27.-a

\section{INTRODUCTION}

The proposed neutrino factory requires muon acceleration to 20 or $50 \mathrm{GeV} / c$ [1]. Since a muon beam has short lifetime of $2.2 \mu \mathrm{s}$ at rest and large emittance of $30 \times$ $10^{-3} \pi \mathrm{m} \mathrm{rad}$ [1], a suitable accelerator has to accelerate muons quickly with large acceptance. An obvious candidate for a muon accelerator is a linear accelerator (linac). However, from a rf power efficiency point of view, a repetitive use of linac with arcs at both ends was suggested; that is known as a recirculating linear accelerator [2]. Another candidate for a muon accelerator with similar advantage in rf power efficiency is a fixed field alternating gradient (FFAG) accelerator. Because of the small orbit shift during acceleration which can fit in a single magnet aperture, a beam can circulate for many turns in a FFAG lattice; thus, the rf power requirement is reduced. Unlike a conventional synchrotron, the repetition rate or acceleration time is determined only by the available rf power with a constant magnetic field.

A scheme of a neutrino factory based on a cascade of muon FFAG accelerators was first proposed in Japan [3]. The FFAG employed in the proposal was a conventional type called a scaling FFAG. A scaling FFAG satisfies the so-called cardinal conditions $[4,5]$ so that the transverse tune is constant in both planes. The magnetic field profile in the radial direction has a $r^{k}$ dependence, where $k$ is a constant field index, and the shape of the orbits is similar for different momenta although the average radius increases as the beam is accelerated. The field profile which

\footnotetext{
*shinji.machida@stfc.ac.uk
}

has a steeper gradient in larger radius region implies that the orbit shift is smaller in the high momentum side.

The constant transverse tune may be a necessary condition for an ordinary accelerator such as a synchrotron because, if there is tune excursion in tune space, a particle will cross resonances which may increase particle amplitude and eventually cause a particle to hit the vacuum chamber. When a FFAG accelerator is used for muon acceleration, however, the constant tune condition may be violated because a beam circulates for only 10 to 20 turns and the amplitude built up with resonance crossing will be harmless. In fact, we showed in a previous paper that a beam was affected not by resonances, but rather by random kicks [6]. A FFAG without the cardinal conditions, and optimized from the point of view of compactness and simplicity, namely with only linear lattice magnets such as dipoles and quadrupoles, is called a nonscaling FFAG [79]. More recent designs of a neutrino factory based on muon acceleration in a FFAG have adopted such a nonscaling type [1].

Although the original purpose of a nonscaling FFAG is muon acceleration, the compact design and simple lattice magnets are attractive characteristics that led to consideration of wider applications. Among them, a particle therapy machine [10] and an accelerator driven system (ADS) [11] are seriously considered as applications of a nonscaling FFAG. In those accelerators, acceleration time is relatively longer, such as a few ms compared with that for muon, which is a few $\mu$ s, because one does not have to compete with particle life time and the rf voltage should be moderate to make the accelerator cost reasonable. However, the slow acceleration rate required for these applications means the problem of resonance crossing becomes an issue 
again. The deterioration of beam quality as a function of errors in a lattice, speed of resonance crossing, etc. should be investigated to ascertain whether such applications are feasible. There were studies on the similar issue such as Aiba et al. [12] which discussed the particle trapping in a nonsystematic nonlinear resonance crossing and Lee et al. [13] which discussed crossing of space charge induced systematic resonances. This paper, however, intended to study the effects of crossing of the lowest order non systematic resonance because alignment errors were inevitable in practice and space charge effects could be reduced with a high machine repetition rate and a lesser number of particles per bunch.

In this paper, we studied amplitude growth of a particle with tracking simulations in a nonscaling FFAG when it was operated with a much slower acceleration rate than that for muons; we considered proton acceleration with moderate rf voltage. First, the results were compared with an analytical relation by Baartman et al. [14,15]. We then calculated the required aperture as a function of errors and acceleration rate. We also studied the effects of nonlinearities which reduce the tune excursion.

\section{SIMULATION SETUP}

\section{A. Accelerator model}

We used the nonscaling FFAG lattice for the electron model for muon acceleration (EMMA) as a simulation base [16]. A proposed nonscaling FFAG for medical use has similar optics although the acceleration rate is much slower [10]. The lattice of EMMA consists of 42 doublet focusing cells. The focusing (QF) and defocusing (QD) quadrupole magnets were shifted in the radial direction to produce a dipole field component. Both transverse tune and the time of flight dependence on momentum were adjusted by changing the field gradients of both QD and QF, and their radial position, with respect to the machine center.

The acceleration was modeled simply by a constant energy increase of a particle at each cell. There are two reasons for this simplification. One is literally its simplicity and the consequent reduction in computational time. The other is, more importantly, to avoid longitudinal and transverse coupling. In a linear nonscaling FFAG without chromaticity correction, the revolution time depends on the transverse amplitude [17]. The large amplitude particle,

TABLE I. EMMA parameters.

\begin{tabular}{ll}
\hline \hline Cell length & $394.481 \mathrm{~mm}$ \\
Number of cell & 42 \\
Focusing & Doublet \\
Quadrupole gradient & \\
QF & $6.7 \mathrm{~T} / \mathrm{m}$ \\
QD & $4.9 \mathrm{~T} / \mathrm{m}$ \\
Injection/extraction momentum & $10.50 / 20.50 \mathrm{MeV} / c$ \\
\hline \hline
\end{tabular}

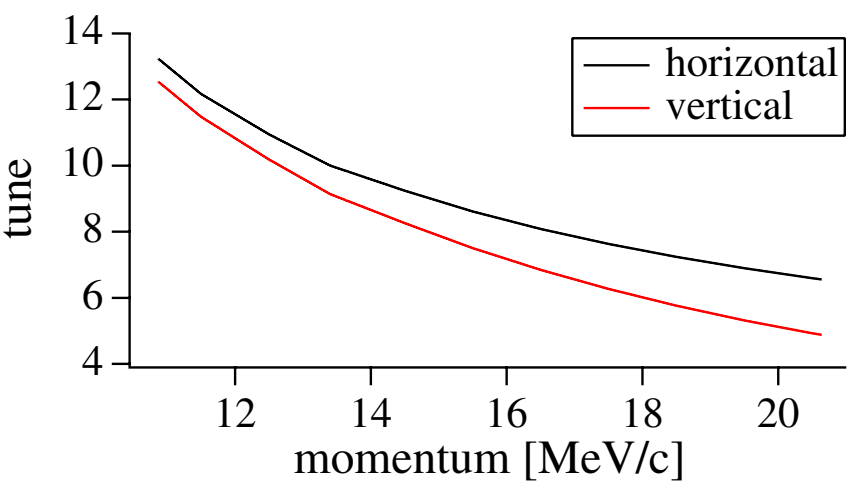

FIG. 1. (Color) Horizontal and vertical total tune as a function of momentum.

for example, takes more time to circulate and that makes energy spread in a bunch. Beyond some certain transverse amplitude, a particle goes to the deceleration phase and is no longer accelerated. In order to separate this problem from the particle loss due to resonances, we applied a constant energy gain independent of transverse amplitude.

The injection and extraction momentum were chosen at 10.75 and $20.75 \mathrm{MeV} / c$, respectively. Although the design values were 10.50 and $20.50 \mathrm{MeV} / c$, respectively, the slight shift of injection momentum in simulation made both the initial horizontal and vertical tune stay away from integer values and therefore resulted in a smaller orbit distortion to start with when alignment errors were included. Table I shows the main parameters of EMMA.

Figure 1 shows horizontal and vertical total tune as a function of momentum. Integer resonances were excited by misalignments of the magnets. Because a dipole component was made with a shift of the quadrupole magnets, the alignment errors of the quadrupoles introduced a nonuniform dipole component around the ring. We assumed that two quadrupole magnets of doublet focusing were on the same table so that QD and QF were misaligned in the same direction and with the same magnitude. There was no assumed misalignment in the longitudinal direction or rotation of magnets.

\section{B. Particle amplitude}

The closed orbit distortion at injection momentum with misalignment errors was first found by iteration, which defined the origin of initial phase space coordinates. Then a particle was launched from the origin. The particle amplitude was, however, measured with respect to the phase space origin which was defined without misalignment errors. The evolution of the amplitude was calculated as

$$
2 J_{y}=\frac{y^{2}+\left(\alpha_{y} y+\beta_{y} y^{\prime}\right)^{2}}{\beta_{y}},
$$

where $\alpha_{y}$ and $\beta_{y}$ are lattice functions of the lattice without 
errors. Although the particle amplitude may be defined with respect to the closed orbit for each individually misaligned lattice, the difference of the amplitude with respect to the phase space origin with and without misalignment errors was small compared with the amplitude growth due to resonances that will be shown later. From a practical point of view, we are interested in how much physical aperture, measured with respect to the center of vacuum chamber, is needed.

The calculated $2 J_{y}$ had high frequency ripples. In order to remove those ripples and show the amplitude behavior relevant to integer tune crossing, $2 J_{y}$ was smoothed with the Savitzky-Golay filter [18].

\section{SIMULATION RESULTS}

\section{A. Single particle behavior}

Figure 2 shows examples of vertical amplitude growth as a function of tune. The amplitude was normalized with momentum $(\beta \gamma)$ so that it stayed constant with acceleration. We will show only the vertical motion in this paper because the horizontal motion is similar and vertical aperture is more restricted than horizontal in practice. A fixed
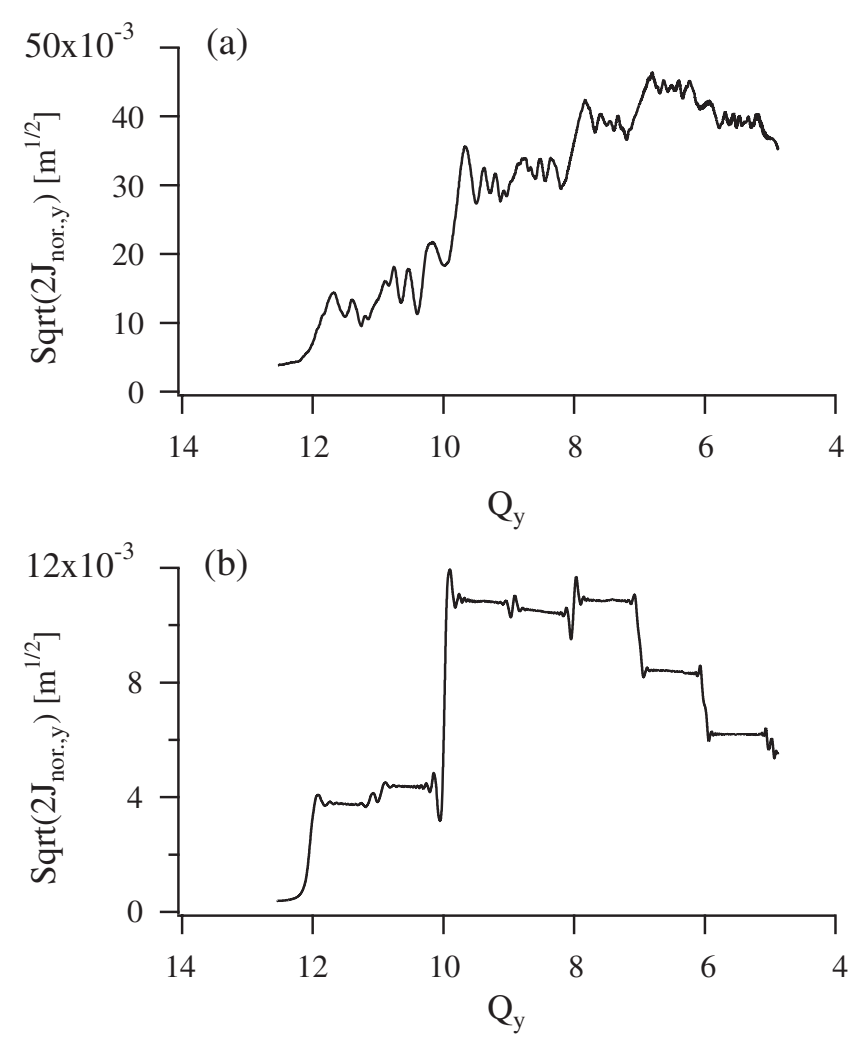

FIG. 2. Examples of single particle normalized amplitude evolution when alignment errors are included: (a) 100 turns to finish the whole cycle, (b) 1000 turns to finish the whole cycle. Note that the magnitude of misalignment in 100 turns acceleration is larger so that the maximum amplitude is larger in (a) than that in (b).

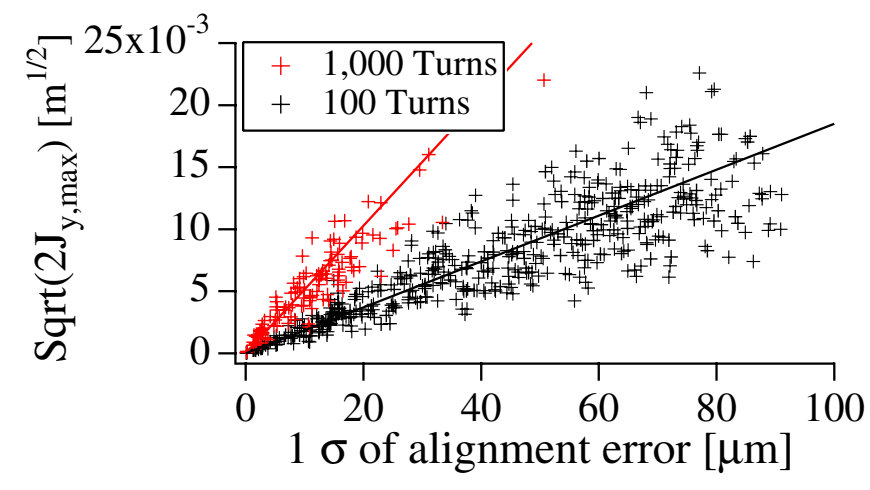

FIG. 3. (Color) Maximum amplitude through the whole cycle for two example acceleration rates of 100 and 1000 turns.

field accelerator always has wider aperture in horizontal plane to accommodate orbit shift due to momentum change. Higher tune was plotted on the left side because the tune decreased when a particle was accelerated. With an acceleration rate of 100 turns to finish the whole cycle, the growth at each integer resonance crossing is already visible as shown in Fig. 2(a). This becomes more obvious when an acceleration rate is slower, for example 1000 turns to finish as shown in Fig. 2(b). Note that the magnitude of misalignment in 100 turns acceleration is larger so that the maximum amplitude is larger in Fig. 2(a) than that in Fig. 2 (b). The finite initial amplitude is due to the definition of the particle amplitude with respect to the phase space origin without misalignment errors as discussed in the previous section. The magnitude is, however, negligible compared with the growth due to the resonance crossing.

We have prepared 501 (enough to see trends) different patterns and amplitudes of alignment errors altogether. The magnitude of the misalignment of 42 doublets was assumed to have a Gaussian distribution with a cut at $2 \sigma$. For each misaligned lattice, a particle was tracked from the closed orbit defined at the injection momentum and the

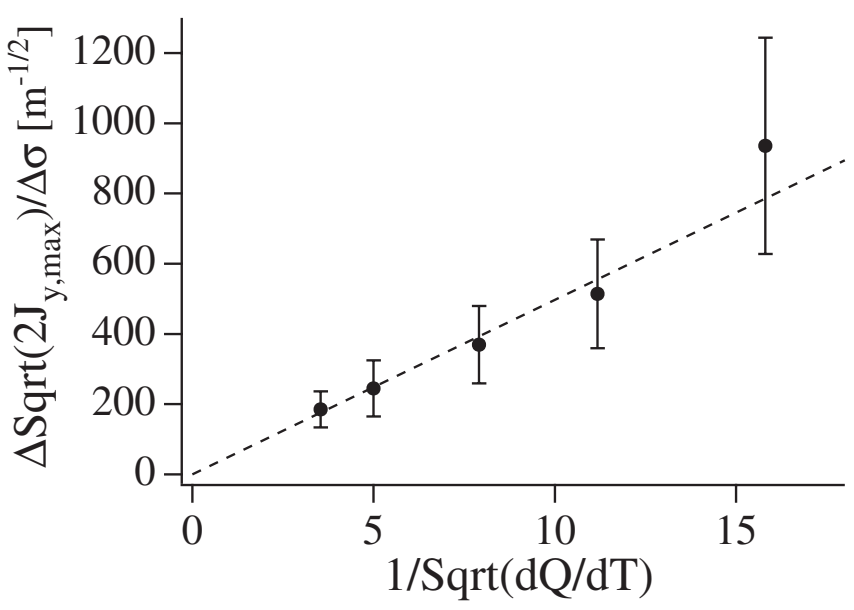

FIG. 4. Amplitude growth as a function of acceleration rate. Error bars indicate rms deviations from the fitted line in Fig. 3. 
maximum value of Eq. (1) through the whole cycle was recorded. Figure 3 shows $\sqrt{2 J_{y, \max }}$ as a function of the alignment errors.

Since $\sqrt{2 J_{y, \max }}$ was more or less proportional to the alignment errors, we fitted the data with a straight line. Together with other acceleration rates: 200, 500, 2000 turns, the gradient of $\sqrt{2 J_{y, \max }}$ as a function of the acceleration rate is depicted in Fig. 4. When the inverse of the square root of a tune change per turn is taken as an abscissa, all the data sit on a straight line. The error bars indicate rms deviations from the fitted line in Fig. 3. To define the tune change per turn, we took the number of integer tune to be crossed, which is 8 , divided by the total number of turns, although it varied from this approximation slightly as Fig. 1 suggests.

\section{B. Dynamic aperture}

The amplitude growth due to integer resonance crossing can put a particle outside the stable region in transverse phase space. We defined the maximum amplitude of survival particles that were accelerated to the final momentum as the dynamic aperture. Initially, a particle had the same $2 J_{x}$ and $2 J_{y}$ and was tracked for the whole acceleration cycle. Figure 5 plots a set of initial particle amplitudes that were NOT accelerated to the final momentum as a function of the alignment error. The step size is $20 \times 10^{-6} \pi \mathrm{m} \mathrm{rad}$ normalized (that is geometrical emittance of about $1 \times$ $\left.10^{-6} \pi \mathrm{m} \mathrm{rad}\right)$. The figure shows that there is no dynamic aperture with alignment errors of $15 \mu \mathrm{m}$ or more when the acceleration takes 1000 turns.

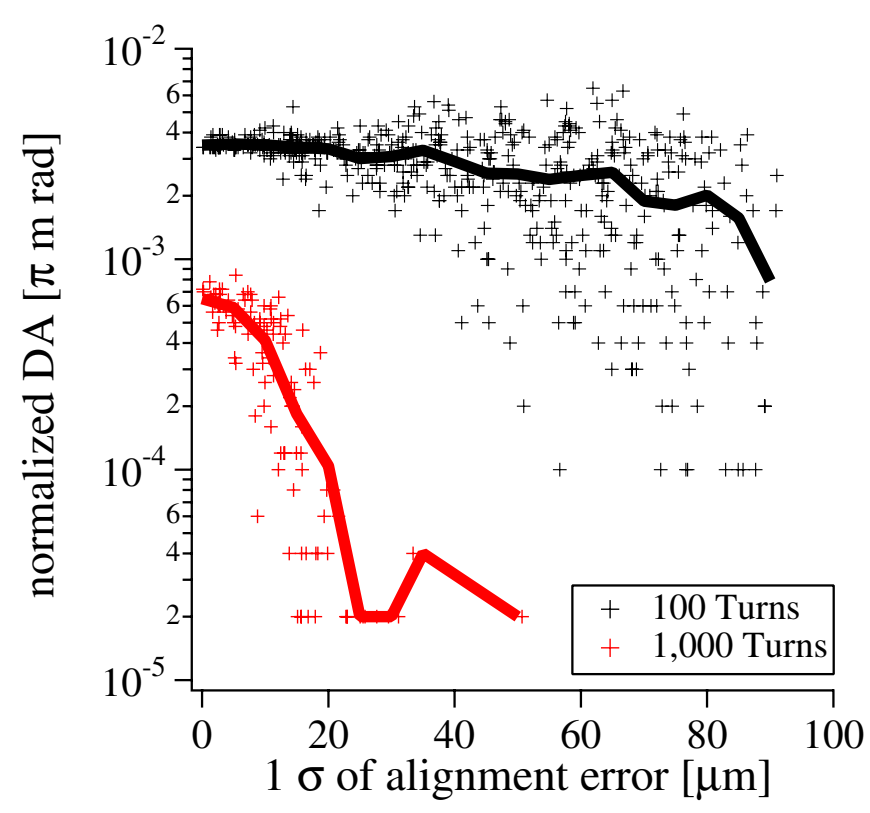

FIG. 5. (Color) Initial amplitude which was not accelerated to the final momentum. Black markers show 100 turns acceleration and red markers show 1000 turns acceleration. Solid curves show the average among the nearby points.

\section{DISCUSSIONS}

\section{A. Amplitude growth}

The simulation results show that the particle amplitude growth due to the integer resonance crossing is proportional to alignment errors and inversely proportional to the square root of the tune change. That indeed agrees with a relation Baartman et al. derived analytically [14,15]:

$$
\Delta A=\frac{\pi}{\sqrt{d Q / d T}} \frac{\bar{R}}{\bar{B}} \frac{B_{n}}{Q},
$$

where $\Delta A$ is the amplitude growth [m], $d Q / d T$ is the tune change per turn, $\bar{R}$ is the average radius of an accelerator $[\mathrm{m}], \bar{B}$ is the average bending field strength $[\mathrm{T}], B_{n}$ is the $n$th harmonic content of error fields [T], and $Q$ is the tune. When $Q=n$, the amplitude growth is excited.

To compare with the simulation results quantitatively, we substituted $\Delta\left(\sqrt{2 J_{y} \beta_{y}}\right)$ for $\Delta A$ with $\beta_{y}=\frac{\bar{R}}{Q}$ and $\frac{d B}{d y} f_{n}$ for $B_{n}$, where $\frac{d B}{d y}$ is the quadrupole strength and $f_{n}$ is the $n$th Fourier component of the alignment errors,

$$
f_{n}=\sum_{m=1}^{m \max } a_{m} \exp (j n m)
$$

The relation finally becomes

$$
\Delta\left(\sqrt{2 J_{y}}\right)=\frac{\pi}{\sqrt{d Q / d T}} \frac{\sqrt{\bar{R}}}{\bar{B}} \frac{(d B / d y) f_{n}}{\sqrt{Q}} .
$$

It is further modified as

$$
\frac{\Delta\left(\sqrt{2 J_{y, \max }}\right)}{\Delta \sigma} / \frac{1}{\sqrt{d Q / d T}}=\frac{\pi \sqrt{\bar{R}}}{\bar{B}} \frac{d B}{d y} \sqrt{n_{\mathrm{res}}}\left\langle\frac{f_{n}}{\Delta \sigma \sqrt{Q}}\right\rangle,
$$

where $n_{\text {res }}$ is the number of integer resonances to be crossed and \langle\rangle takes the average. We assumed that the maximum amplitude after $n_{\text {res }}$ crossing was $\sqrt{n_{\text {res }}}$ times the growth per crossing because there should be no correlation in the betatron phases every time a particle received the growth. Using the following values,

$$
\begin{aligned}
& \bar{R}=2.5[\mathrm{~m}], \\
& \bar{B}=\frac{B \rho}{\bar{R}}=0.020[\mathrm{~T}], \\
& \frac{d B}{d y}=\left(\frac{d B}{d y}\right)_{\mathrm{QF}}+\left(\frac{d B}{d y}\right)_{\mathrm{QD}}=6.7-4.9=1.8[\mathrm{~T} / \mathrm{m}], \\
& n_{\mathrm{res}}=8, \text { and } \\
&\left\langle\frac{f_{n}}{\Delta \sigma \sqrt{Q}}\right\rangle \text { from the } 501 \text { different error patterns, }
\end{aligned}
$$


the right-hand side of the equation (5) becomes $61 \mathrm{~m}^{-1 / 2}$. The gradient of Fig. 4, on the other hand, indicates $50 \mathrm{~m}^{-1 / 2}$. Taking account of the adiabatic damping of the amplitude, which in fact reduces the maximum amplitude in simulation less than $\sqrt{n_{\text {res }}}$ times the growth (but the reduction rate is no less than $1 / \sqrt{2}$ where 2 is the initial and final momentum ratio), the agreement between the simulation results and the formula is good.

\section{B. Chromaticity correction}

The rapid decrease of the dynamic aperture with alignment errors in Fig. 5 implies that a nonscaling FFAG does not work with an acceleration rate of order 1000 turns. A few $\mu \mathrm{m}$ alignment tolerance is somewhat beyond the level we can achieve practically, which is a few times $10 \mu \mathrm{m}$. If the reduction of dynamic aperture is solely because of the resonance crossing, a smaller total tune excursion, ideally within neighboring integers, should improve the dynamic aperture. On the other hand, there is a concern that introducing additional nonlinearities to correct the chromaticity may reduce the dynamic aperture even without alignment errors.

Sextupole and octupole components were added to the quadrupoles for chromaticity correction. To minimize the required strength of these components, the center of both multipoles was located at the orbit of the final momentum and we fixed the tune around the one which was determined by the final momentum. The strength of sextupoles and octupoles was obtained with the downhill simplex method [19]. Figure 6 is the resulting tune excursion. The horizontal tune is located just between integer of 6 and 7 , but the vertical tune starts from above integer 5 and decreases to below 5 .

With these multipole configurations, the dynamic aperture was surveyed as a function of alignment errors. As green markers in Fig. 7 show, dynamic aperture was reduced. It is obvious that the dynamic aperture even without error was reduced because of additional nonlinearities.

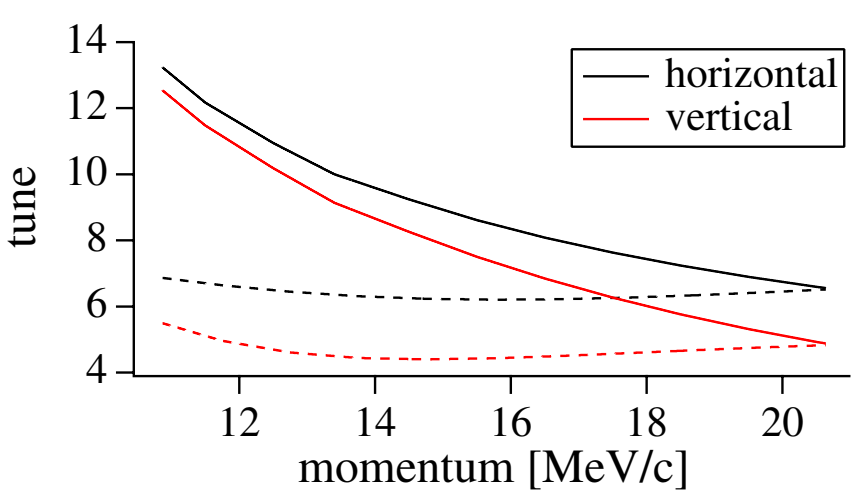

FIG. 6. (Color) Dashed lines show horizontal and vertical total tunes as a function of momentum when sextupole and octupole are introduced to correct the chromaticity. Solid lines are the same as Fig. 1.

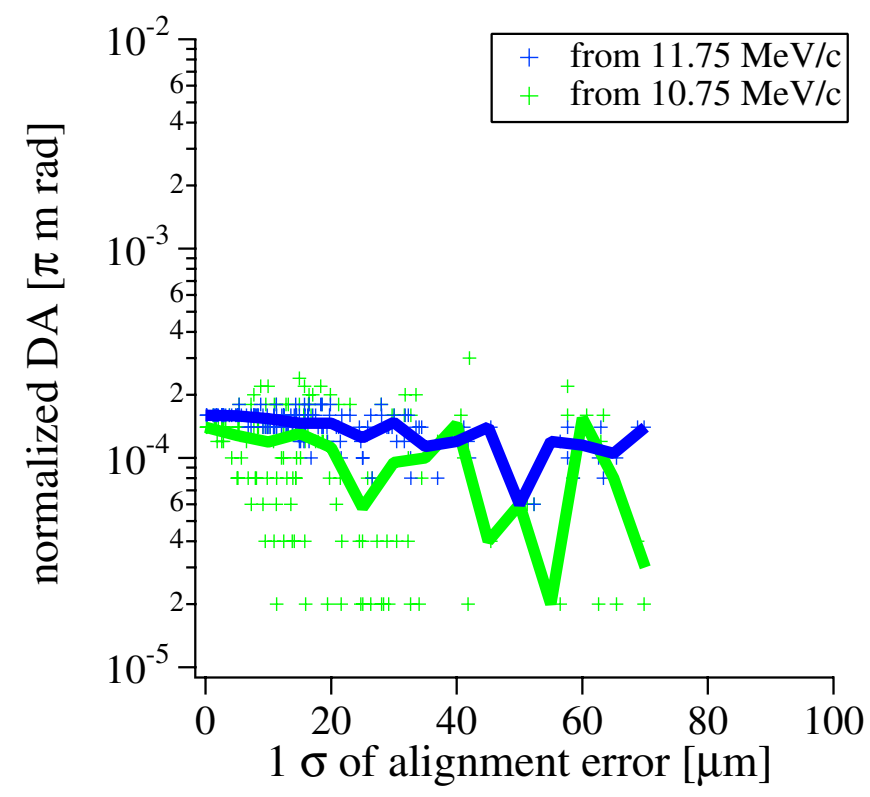

FIG. 7. (Color) Initial amplitude which was not accelerated to the final momentum. Lattice has chromaticity correction with sextupole and octupole. It takes 1000 turns to finish the whole cycle. Green markers show the case when acceleration started from $10.75 \mathrm{MeV} / c$. Blue markers show the case when acceleration started from $11.75 \mathrm{MeV} / c$ to avoid $Q y=5$. Solid curves show the average among the nearby points.

Furthermore, the resonance crossing at $Q y=5$ became the major source of amplitude growth as shown in Fig. 8.

In order to avoid the integer resonance crossing at all, we started the particle tracking from $11.75 \mathrm{MeV} / c$. Although this does not solve the problem in a real machine operation, it is interesting to see how the integer resonance crossing affects the beam. Blue markers in Fig. 7 show that the dynamic aperture is less sensitive to the alignment errors although the maximum aperture with an error-free lattice does not change. It clearly shows that, if the required normalized beam acceptance is $100 \times 10^{-6} \pi \mathrm{m} \mathrm{rad}$ (geo-

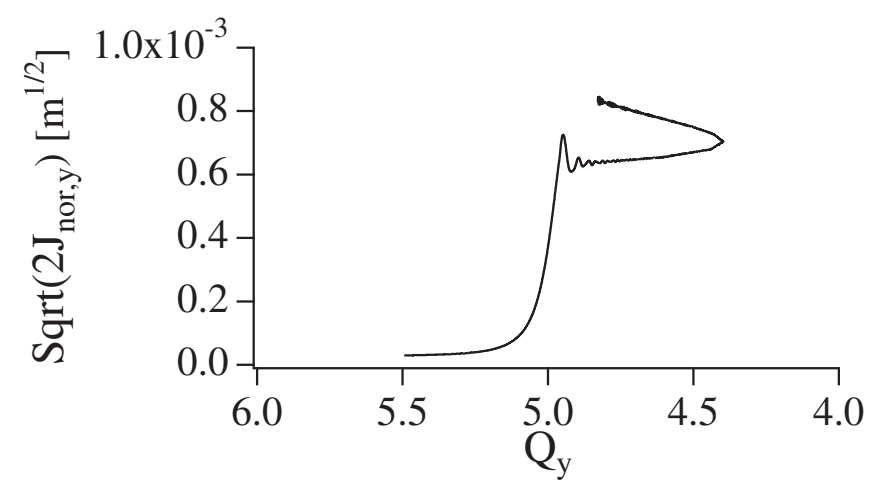

FIG. 8. Example of single particle normalized amplitude evolution when alignment errors are included. It takes 1000 turns to finish the whole cycle. As Fig. 6 indicates, the vertical tune starts from around 5.5 and goes to the tune of around 4.3. Then, it increases again. 
metrical acceptance of about $5 \times 10^{-6} \pi \mathrm{m} \mathrm{rad}$ ) or less, a nonscaling FFAG may be used even with a slow acceleration rate of 1000 turns with chromaticity correction. In other words, chromaticity correction is an essential ingredient to operate a nonscaling FFAG with practical alignment errors.

Lastly, we should make a comment on the elimination of the last integer resonance crossing that occurs when accelerating from the original momentum of $10.75 \mathrm{MeV} / c$. To accelerate from this momentum, further adjustment of the quadrupoles, sextupoles, and octupoles is necessary such that both the transverse tunes stay between neighboring integers throughout the cycle. This should be investigated. An alternative approach is to reduce the tune variation by introducing a wedge-shaped quadrupole, as proposed by Johnstone [20]. This approach could be solely used or combined with nonlinear multipoles. Another remedy for the problem would be harmonic correction with fixed field correction magnets. The idea of resonance correction cannot be applied in a nonscaling FFAG in general because it is not possible to correct several harmonics at once with fixed field magnets [6]. If there is, however, only a single harmonic left to be corrected, it should be possible to reduce the harmonic with the conventional correction algorithm such as the harmonic correction scheme and the $\chi^{2}$-minimization method [21].

\section{SUMMARY}

With particle tracking simulations, the effects of resonance crossing in a linear nonscaling FFAG were studied when there were alignment errors and when an accelerator was operated with a relatively slow acceleration rate of 100 or 1000 turns. When there were practical errors such as a few times $10 \mu \mathrm{m}$ rms misalignment, the resonance behavior started appearing with 100 turns operation and became clear with 1000 turns operation. In the latter case, dynamic aperture practically disappeared. It was shown that the amplitude growth dependence on acceleration rate and alignment error agreed with the analytical formula by Baartman et al.

Chromaticity correction with sextupoles and octupoles was applied to eliminate resonance crossing. As a result, the maximum available dynamic aperture in a lattice without errors was reduced as expected. On the other hand, the machine became less sensitive to misalignment errors. Although it does not have enough aperture to be suitable as a muon accelerator any more, for some applications where the large acceptance is not a high priority demand (such as a proton driver and a particle therapy machine), chromaticity correction seems to be an essential ingredient to make a nonscaling FFAG work.

\section{ACKNOWLEDGMENTS}

The work is supported by the UK Neutrino Factory/ Particle Physics and Astronomy Research Council (PPARC) under Contract No. 2054. We would like to thank Dr. C.R. Prior and Dr. G. H. Rees for their continuous encouragement, and Dr. S. Ohnuma, Dr. D. J. Kelliher, and Mr. D. C. Plostinar for their helpful comments on the manuscript.

[1] http://www.cap.bnl.gov/mumu/project/ISS/ISSAcceleratorWG-final.pdf.

[2] S. A. Bogacz and V. A. Lebedev, Nucl. Instrum. Methods Phys. Res., Sect. A 503, 306 (2003).

[3] S. Machida, Nucl. Instrum. Methods Phys. Res., Sect. A 503, 41 (2003).

[4] K. R. Symon, D. W. Kerst, L. W. Jones, L. J. Laslett, and K. M. Terwillinger, Phys. Rev. 103, 1837 (1956).

[5] A. A. Kolomensky and A. N. Lebedev, Theory of Cyclic Accelerators (North-Holland, Amsterdam, 1966), p. 337.

[6] S. Machida and D. J. Kelliher, Phys. Rev. ST Accel. Beams 10, 114001 (2007).

[7] C. Johnstone, W. Wan, and A. Garren, in Proceedings of the Particle Accelerator Conference, New York, 1999 (IEEE, New York, 1999), p. 3068, http://www.jacow.org.

[8] E. Keil and A. M. Sessler, Nucl. Instrum. Methods Phys. Res., Sect. A 538, 159 (2005).

[9] D. Trbojevic, E. D. Courant, and M. Blaskiewicz, Phys. Rev. ST Accel. Beams 8, 050101 (2005).

[10] E. Keil, A. M. Sessler, and D. Trbojevic, Phys. Rev. ST Accel. Beams 10, 054701 (2007).

[11] M. Tanigaki, Y. Mori, M. Inoue, K. Mishima, S. Shiroya, Y. Ishi, S. Fukumoto, and S. Machida, in Proceedings of the 10th European Particle Accelerator Conference, Edinburgh, Scotland, 2006 (EPS-AG, Edinburgh, Scotland, 2006), p. 2367, http://www.jacow.org.

[12] M. Aiba, S. Machida, Y. Mori, and S. Ohnuma, Phys. Rev. ST Accel. Beams 9, 084001 (2006).

[13] S. Y. Lee, G. Franchetti, I. Hofmann, F. Wang, and L. Yang, New J. Phys. 8, 291 (2006).

[14] R. Baartman, http://www.triumf.ca/ffag2004/.

[15] G. Guignard, CERN Report No. CERN 77-10, 1977.

[16] S. Machida, AIP Conf. Proc. 981, 122 (2008).

[17] S. Machida, Phys. Rev. ST Accel. Beams 9, 104002 (2006).

[18] W.H. Press, S. A. Teukolsky, W. T. Vetterling, and B.P. Flannery, Numerical Recipes in FORTRAN (Cambridge University Press, Cambridge, England, 1992), p. 644.

[19] See Ref. [18], p. 402.

[20] C. Johnstone and S. Koscielniak, in Proceedings of the 2007 Particle Accelerator Conference, Albuquerque, New Mexico, 2007 (IEEE, Albuquerque, New Mexico, 2007), p. 2951, http://www.jacow.org.

[21] S. Y. Lee, Accelerator Physics (World Scientific, Singapore, 1999), p. 81. 